\title{
Association between statin-associated myopathy and skeletal muscle damage
}

\author{
Markus G. Mohaupt MD, Richard H. Karas MD PhD, Eduard B. Babiychuk PhD, \\ Verónica Sanchez-Freire, Katia Monastyrskaya PhD, Lakshmanan lyer PhD, Hans Hoppeler MD, \\ Fabio Breil, Annette Draeger MD
}

An abridged version of this article appeared in the July 7, 2009, issue of CMAJ.

\section{ABSTRACT}

Background: Many patients taking statins often complain of muscle pain and weakness. The extent to which muscle pain reflects muscle injury is unknown.

Methods: We obtained biopsy samples from the vastus lateralis muscle of 83 patients. Of the 44 patients with clinically diagnosed statin-associated myopathy, 29 were currently taking a statin, and 15 had discontinued statin therapy before the biopsy (minimal duration of discontinuation 3 weeks). We also included 19 patients who were taking statins and had no myopathy, and 20 patients who had never taken statins and had no myopathy. We classified the muscles as injured if $2 \%$ or more of the muscle fibres in a biopsy sample showed damage. Using reverse transcriptase polymerase chain reaction, we evaluated the expression levels of candidate genes potentially related to myocyte injury.

Results: Muscle injury was observed in 25 (of 44) patients with myopathy and in 1 patient without myopathy. Only 1 patient with structural injury had a circulating level of creatine phosphokinase that was elevated more than $1950 \mathrm{U} / \mathrm{L}$ (10X the upper limit of normal). Expression of ryanodine receptor 3 was significantly upregulated in patients with biopsy evidence of structural damage (1.7, standard error of the mean 0.3).

Interpretation: Persistent myopathy in patients taking statins reflects structural muscle damage. A lack of elevated levels of circulating creatine phosphokinase does not rule out structural muscle injury. Upregulation of the expression of ryanodine receptor 3 is suggestive of an intracellular calcium leak.

Une version française de ce résumé est disponible à l'adresse www.cmaj.ca/cgi/content/full/cmaj.081785/DC1

CMAJ 2009;181(1-2):E11-E18 tatins are among the most widely prescribed medications worldwide. Although their overall safety profile is excellent, myalgia without functional muscle impairment commonly affects patients taking statins. The clinical manifestations of statin-associated myopathy include pain and muscle weakness. Observational studies have shown a myalgia rate of $10 \%-15 \%$ among patients taking statins. ${ }^{1,2}$ Fulminant and potentially fatal rhabdomyolysis may also occur. Myalgia is typically considered by patients and physicians to be a minor adverse effect. ${ }^{3-5}$ Current consensus guideline support continuation of the statin therapy as long as circulating levels of creatine phosphokinase are less than $1950 \mathrm{U} / \mathrm{L}$ (10× the upper limit of normal) ${ }^{6}$

We sought to determine whether statin-associated myopathy is associated with underlying structural muscle damage. We investigated whether the extent of muscle damage is reflected by the level of circulating creatine phosphokinase. We also sought to identify alterations in the expression of genes expressed in myocytes, which could provide insight into the cause of statin-associated myopathy.

\section{Methods}

\section{Patients}

Samples of the vastus lateralis muscle were collected from 83 people in 5 groups. The first control group comprised 10 healthy male volunteers who had never taken statins and who had no complaints of muscle pain (biopsy samples from these patients were obtained for a previous study ${ }^{7}$ ). The second control group included 10 patients who were age-matched to patients in the myopathy group. These patients were each receiving treatment for a condition not related to their muscles. These patients were recruited from a pool of patients in the Department of Nephrology and Hypertension, and they had no history of statin use or muscle complaints. Several patients in this group had hypercholesterolemia but had never taken statins. Biopsy samples were obtained from these patients before they began statin therapy as part of an ongoing longitudinal study.

The third group comprised 15 patients with clinically diagnosed statin-associated myopathy who had discontinued statin therapy (discontinued for a minimum of 3 [median 12] weeks

From the Department of Nephrology and Hypertension (Mohaupt), Inselspital, University of Bern, Bern, Switzerland; the Molecular Cardiology Research Institute and Division of Cardiology (Karas, lyer), Tufts-New England Medical Center, Boston, USA; and the Institute of Anatomy (Babiychuk, Sanchez-Freire, Monastyrskaya, Hoppeler, Breil, Draeger), University of Bern, Bern, Switzerland 
before biopsy). These patients were referred to the Department of Nephrology and Hypertension for alternative treatment options for hypercholesterinemia because they refused to continue statin therapy. These patients reported persistent myalgia or muscle weakness, or both, in the absence of other reasons for muscle disease.

The fourth group included 29 patients who had a history of clinically diagnosed statin-associated myopathy and who were receiving statin therapy at the time of the biopsy. These patients were recruited from the pool of patients in the Department of Nephrology and Hypertension

The fifth group included 19 patients who had received statin therapy for hypercholesterolemia for 4-20 years and were taking statins at the time of the biopsy. These patients had no muscle complaints.

Patients were identified as having statin-associated myopathy by clinical criteria consistent with the recommendations of the Muscle Safety Expert Panel. ${ }^{3}$ These patients had persistent complaints of myalgia, cramps or muscle weakness predominately in the trunk or proximal muscle groups. Some reported that their pain worsened with exertion while taking statins and in the absence of other reasons for myopathy. Some patients had elevated serum concentrations of creatine phosphokinase (585-975 U/L [3-5× the upper limit of normal]).

Patients with myopathy (with or without statin use) were referred to the Department of Nephrology and Hypertension by local hospitals or physicians for management of their muscle symptoms. The diagnosis of statin-associated myopathy and decisions about the continued use of statins were made by the clinical team caring for each patient. Patients taking statins without muscle complaints were recruited via advertisements in local newspapers.

The vastus lateralis muscle was biopsied at midthigh level by use of the technique of Bergström. ${ }^{8}$ The samples were processed within 10 minutes after excision. Although the vastus lateralis muscle is rarely affected by myalgia, it serves as a reference muscle and is routinely biopsied when systemic muscle pathology is investigated.

This study was conducted with the approval of the ethics committee of the Canton of Bern Switzerland. All patients gave informed consent.

\section{Tissue processing}

An average of 3 tissue blocks (about $3 \mathrm{~mm} \smile 6 \mathrm{~mm}$ ) per patient were fixed and processed for semi-thin sectioning and ultrastructural analysis by electron microscopy. All samples were fixed in $6.25 \%$ glutaraldehyde solution and embedded in epoxy resin. ${ }^{9}$

\section{Immunohistochemistry and ultracryomicrotomy}

One or more cylinders of some tissue samples were fixed in 4\% paraformaldehyde and processed for ultra-thin frozen sectioning. ${ }^{10}$ We labelled the $\mathrm{T}$ tubules with a monoclonal antibody against annexin A6 and a secondary polyclonal antibody coupled to $\mathrm{Cy} 3 .{ }^{10}$ We viewed the T tubules using a ZEISS Axiophot 2 fluorescence microscope, and we captured photomicrographs using a digital CCD camera (Ultrapix, Astrocam).

\section{RNA isolation and gene expression}

We isolated total RNA from muscle biopsy samples using the RNeasy Fibrous Tissue Kit (Qiagen), according to the manufacturer's instructions. The samples were treated with DNase I (Qiagen) to avoid genomic DNA contamination. We measured the total RNA concentration by ultraviolet spectrophotometry at $260 \mathrm{~nm}$ (DU 530, Beckman Coulter). The purity of the RNA was determined by the ratio of the absorbance at $260 \mathrm{~nm}$ to the absorbance at $280 \mathrm{~nm}$ and confirmed by agarose gel electrophoresis. We synthesized cDNA using random hexamer primers and the High Capacity cDNA Reverse Transcription Kit (Applied Biosystems).

We analyzed the expression of the following 8 genes that code for proteins located in the T-tubule membrane and adjacent sarcoplasmic reticulum and that are involved in the regulation of intracellular calcium homeostasis: Inositol 1,4,5triphosphate receptor, type 1 (ITPR1-Hs00181881_m1); inositol 1,4,5-triphosphate receptor, type 2 (ITPR2Hs00181916_m1); inositol 1,4,5-triphosphate receptor, type 3 (ITPR3-Hs00609908_m1); ryanodine receptor 1 (RYR1Hs00166991_m1); ryanodine receptor 3 (RYR3Hs00168821_m1); sarco-endoplasmic reticulum transporting $\mathrm{Ca}^{2+}$ ATPase 1 (ATP2A1-Hs00188877_m1); sarco-endoplasmic reticulum transporting $\mathrm{Ca}^{2+}$ ATPase 2 (ATP2A2Hs00544877_m1); and sarco-endoplasmic reticulum transporting $\mathrm{Ca}^{2+}$ ATPase 3 (ATP2A3-Hs00193090_m1).

We performed expression studies using validated TaqMan Gene Expression Assays with 18S rRNA as a reference gene. The reactions were performed using the 7900HT Fast RealTime PCR System (Applied Biosystems). The default program was used (40 cycles, each consisting of 15 seconds at $95^{\circ} \mathrm{C}$ followed by 1 minute at $60^{\circ} \mathrm{C}$ ). Data acquisition was performed according to the manufacturer's instructions.

For analysis of the expression data, we carried out a relative quantity study. The expression values of the target genes were normalized to the concentration of $18 \mathrm{~S}$ rRNA. We calculated gene expression values based on the comparative threshold cycle $(\mathrm{Ct})$ method. The threshold cycle for each gene and 18S rRNA in each sample was determined and used to calculate $\mathrm{Ct}$ values. The $\mathrm{Ct}$ values were calculated by subtracting the $\mathrm{Ct}$ of the calibrator from the $\mathrm{Ct}$ value of each target $\left(\mathrm{Ct}=\mathrm{Ct}_{\text {gene }}-\mathrm{Ct}_{18 \mathrm{~S} \mathrm{rRNA}}\right)$. The relative quantities were calculated with the equation: relative quantity $=2^{-\mathrm{Ct}}$. To calculate the relative quantity, we designated as calibrators the average $\mathrm{Ct}$ values from the group without structural damage. All gene amplification reactions were performed in triplicate.

\section{Tissue analysis}

An observer (A.D.) skilled in light and electron microscopy evaluated the specimens. The observer was unaware of the patient groups. The number of muscle fibres with structural abnormalities in each section was determined. Significant injury was defined as $2 \%$ or more damaged fibres per biopsy sample. This arbitrary value reflected what we considered to be a clinically meaningful level of damage. Smaller amounts of damage may be found in patients with no symptoms of myopathy. 


\section{Statistical analysis}

The data are expressed as mean and standard error of the mean (SEM). We compared the prevalence of muscle injury among the groups by the use of the Fisher exact test. We performed multiple group comparisons for categorical variables by nonparametric analysis of variance by ranks. For continuous variables, we performed analysis of variance followed by the Dunn Multiple Comparison Test. We evaluated the corre- lation between the circulating level of creatine phosphokinase and the extent of injury using the Pearson correlation coefficient. For mRNA expression analysis, normality was evaluated with the Kolmogorov-Smirnov test $(\alpha=0.05)$, with the Lilliefors significance correction. Because the expression of the genes did not have a normal distribution, we performed a Mann-Whitney $U$ test. The level of significance was set at $p<0.05$.

Table 1: Characteristics of patients included in a study of the relation between statin-associated myopathy and structural muscle damage

\begin{tabular}{|c|c|c|c|c|c|}
\hline \multirow[b]{2}{*}{ Characteristic } & \multicolumn{5}{|c|}{ No. $(\%) *$ of patients } \\
\hline & $\begin{array}{c}\text { Control } \\
\text { group } 1 \dagger \\
n=10\end{array}$ & $\begin{array}{c}\text { Control } \\
\text { group } 2 \ddagger \\
n=10\end{array}$ & $\begin{array}{l}\text { Former statin users } \\
\text { with myopathy } \\
\qquad n=15\end{array}$ & $\begin{array}{l}\text { Current statin users } \\
\text { with myopathy } \\
n=29\end{array}$ & $\begin{array}{l}\text { Current statin users } \\
\text { with no myopathy } \\
\qquad n=19\end{array}$ \\
\hline Female, \% & 0 & 30 & 47 & 24 & 37 \\
\hline Age, yr, mean (range) & $26(23-29)$ & $57(41-74)$ & $59(34-73)$ & $54(34-76)$ & $66(49-84)$ \\
\hline $\begin{array}{l}\text { Total cholesterol, } \mathrm{mmol} / \mathrm{L} \text {, median } \\
\text { (IQR) }\end{array}$ & ND & $6.2(2.34)$ & $6.5(2.70)$ & $5.0(1.12)$ & $5.4(1.44)$ \\
\hline $\mathrm{HDL}, \mathrm{mmol} / \mathrm{L}$, median (IQR) & ND & $1.84(0.89)$ & $1.58(0.70)$ & $1.6(0.58)$ & $1.5(0.64)$ \\
\hline Triglycerides, mmol/L, median (IQR) & ND & $2.62(2.09)$ & $2.5(5.34)$ & $2.45(3.35)$ & $2.1(1.79)$ \\
\hline LDL, mmol/L, median (IQR) & ND & $4.2(0.90)$ & $5.15(3.68)$ & $2.9(1.10)$ & $3.2(1.30)$ \\
\hline \multicolumn{6}{|l|}{ CPK, U/L } \\
\hline Median (IQR) & ND & $110(250)$ & $114(117)$ & $118(424)$ & $109(44.5)$ \\
\hline > 975 U/L (5× ULN) & ND & $0(0)$ & $0(0)$ & $4(14)$ & $0(0)$ \\
\hline$>1950$ U/L $(10 \times$ ULN $)$ & ND & $0(0)$ & $0(0)$ & $1(3)$ & $0(0)$ \\
\hline \multicolumn{6}{|l|}{ Active statin use } \\
\hline Atorvastatin & $0(0)$ & $0(0)$ & $0(0)$ & $5(17)$ & $0(0)$ \\
\hline Simvastatin & $0(0)$ & $0(0)$ & $0(0)$ & $12(41)$ & $15(74)$ \\
\hline Fluvastatin & $0(0)$ & $0(0)$ & $0(0)$ & $2(7)$ & $0(0)$ \\
\hline Pravastatin & $0(0)$ & $0(0)$ & $0(0)$ & $9(31)$ & $4(21)$ \\
\hline Rosuvastatin & $0(0)$ & $0(0)$ & $0(0)$ & $1(3)$ & $0(0)$ \\
\hline \multicolumn{6}{|l|}{ Previous statin use } \\
\hline Atorvastatin & $0(0)$ & $0(0)$ & $6(40)$ & $6(21)$ & $0(0)$ \\
\hline Simvastatin & $0(0)$ & $0(0)$ & $8(53)$ & $2(7)$ & $1(5)$ \\
\hline Fluvastatin & $0(0)$ & $0(0)$ & $1(7)$ & $2(7)$ & $0(0)$ \\
\hline Pravastatin & $0(0)$ & $0(0)$ & $8(53)$ & $5(17)$ & $0(0)$ \\
\hline Cerivastatin & $0(0)$ & $0(0)$ & $1(7)$ & $0(0)$ & $0(0)$ \\
\hline $\begin{array}{l}\text { No. (\%) of muscle fibres damaged; } \\
\text { [range, \%] }\end{array}$ & $0(0)$ & $0(0)$ & $9(60)[2.8-100]$ & $9(60)$ [3.3-43] & $1(5)$ \\
\hline \multicolumn{6}{|l|}{ Concomitant medication use } \\
\hline Fibrates & 0 & $0(0)$ & $0(0)$ & $0(0)$ & None \\
\hline Immunosuppressants & 0 & $2(20)$ & $5(33)$ & $14(48)$ & None \\
\hline Corticosteroids & 0 & $3(33)$ & $5(33)$ & $9(31)$ & None \\
\hline Warfarin & 0 & $0(0)$ & $1(7)$ & $1(3)$ & None \\
\hline Macrolid antibiotics & 0 & $1(11)$ & $1(7)$ & $2(7)$ & None \\
\hline Antifungals & & $0(0)$ & $0(0)$ & $0(0)$ & None \\
\hline HIV-protease inhibitors & 0 & $1(11)$ & $0(0)$ & $0(0)$ & None \\
\hline
\end{tabular}

Note: $\mathrm{CPK}=$ creatine phosphokinase, $\mathrm{HDL}=$ high-density lipoprotein, IQR = interquartile range, LDL = low-density lipoprotein, $\mathrm{nd}=$ not determined, $\mathrm{ULN}=$ upper limit of normal.

*Unless otherwise indicated

tPatients with no history of statin use or muscle complaints.

‡Patients with no history of statin use or muscle complaints. These patients were age-matched to patients with myopathy. 


\section{Results}

Most of the patients with statin-associated myopathy were men with moderate hypercholesterolemia (Table 1). The average age was 56.5 years. Simvastatin and pravastatin were the most commonly reported statins used, and $25 \%$ of patients had taken more than 1 statin.

The risk of developing statin-associated myopathy has been reported to be exacerbated by a number of drugs that interfere with the metabolism of statins. ${ }^{11-14}$ Because patients in our study were middle-aged and had multiple comorbidities, most were taking several drugs. About $40 \%$ of all patients with myopathy were taking corticosteroids or immunosuppressants, or both. Therefore, we include an age-matched control group of 10 patients who were not taking statins but were taking other medications, which were also matched to those in the myopathy group (Table 1).

\section{Myopathy}

Patients with myopathy reported weakness that was generally slight. They reported having difficulty rising from a chair without arm support. Muscle pain was reported predominately in the trunk and proximal muscle groups and was exacerbated by exercise. Patients reported that their symptoms were severe enough to interfere with the activities of daily living and that they had decreased exercise capacity. The symptoms had lasted for several weeks. Typically, the symptoms disappeared within days after cessation of statin therapy. In 3 cases, the symptoms persisted after statin therapy had been discontinued for more than 1 month (Table 2). One patient, who presented with overt rhabdomyolysis (creatine phosphokinase $57657 \mathrm{U} / \mathrm{L}$ ), required admission to hospital for the management of muscle pain.

\section{Muscle injury}

Significant muscle injury was observed among patients with myopathy and in 1 patient who was taking long-term statin therapy and who had no myopathy (Table 1). There was no significant damage in the fibres of control patients not taking statins. Significant muscle damage was observed in 9 of 15

Table 2: History of myopathy among patients who used statins

\begin{tabular}{lcc}
\hline & \multicolumn{2}{c}{ No. (\%)* patients } \\
\cline { 2 - 3 } Symptom & $\begin{array}{c}\text { Former statin users } \\
\text { with myopathy }\end{array}$ & $\begin{array}{c}\text { Current statin users } \\
\text { with myopathy }\end{array}$ \\
\hline Myalgiat & $10(67)$ & $14(48)$ \\
$\begin{array}{l}\text { Weakness in proximal limb muscle } \\
\text { and trunk }\end{array}$ & $3(20)$ & $11(38)$ \\
\hline $\begin{array}{l}\text { Cramps } \\
\text { No. of weeks since discontinuing } \\
\text { statin use, median [range] }\end{array}$ & $2(13)$ & $2(7)$ \\
$\begin{array}{l}\text { Continuation of symptoms after } \\
\text { statin withdrawal for }>1 \text { month }\end{array}$ & $3(20)$ & NA \\
\hline
\end{tabular}

Note: NA = not applicable.

*Unless otherwise indicated.

†Predominantly in the muscles of the proximal limbs and exacerbated with exercise.

‡Myalgia, weakness or cramps. patients with myopathy who had discontinued statin therapy and in 16 of 29 patients with myopathy currently receiving statin therapy (Table 1). Compared to control patients, those who currently $(p<0.001)$ or previously $(p<0.001)$ used statins had significantly more muscle damage. There was no significant difference in the amount of damage between patients currently and formerly taking statins $(p=0.90)$.

We found lesions in $3.3 \%-43 \%$ of the muscle fibres in the injured skeletal muscles from patients with myopathy currently taking statins and in $2.8 \%-100 \%$ of the muscle fibres from patients with myopathy who were former statin users (Table 1). In the injured muscles of patients with myopathy, the median percent of injured fibres was $9.5 \%(25 \%-75 \%$ confidence interval [CI] 5.1\%-17.6\%) among current statins users and $9.0 \%$ (25\%-75\% CI 3.3\%-19.1\%) among former statin users.

Of the 9 patients with significant muscle injury, 6 had stopped taking stains within 5-20 weeks, and 3 had stopped between 1 year and more than 5 years (median 12 weeks). The severity of muscle damage was not linearly correlated with length of statin therapy.

\section{Electron microscopy}

Although damaged muscle fibres were widespread in most biopsy samples, the degree of destruction in individual fibres was modest. Representative images from the muscle biopsy samples from patients who had or had not taken statins are shown in Figure 1. Light microscopy of semi-thin sections showed subsarcolemmal detachment of the contractile apparatus (Figure 1A, Figure 1B). Electron microscopy confirmed this finding and showed that the sarcolemma was undamaged (Figure 1C). Occasionally, we observed ghost fibres, which are hollow sarcolemmal tubes of degenerated cells (Figure 1D). There was marked variations in the size of the fibres among patients with myopathy (Figure 1E, Figure 1F).

On examination of the semi-thin sections, we found extensive intracellular vacuolization in skeletal muscle from a patient with myopathy currently taking statins (Figure 1G) and a patient with myopathy who had discontinued statin therapy (Figure $1 \mathrm{H}$ ). The muscle fibres from patients in the control groups showed no vacuolization (Figure 1I, Figure 1J).

The intracellular vacuoles correspond to membranous cavities, as shown by electron microscopy (Figure $1 \mathrm{~K}$ and Figure $1 \mathrm{~L})$. Labelling with an antibody against annexin A6, a T-tubular marker protein, showed that the distribution of these vacuoles was consistent with the $\mathrm{T}$ tubule system ${ }^{10}$ (Figure 2).

\section{Gene expression}

We focused our gene expression analysis on the expression of genes that code for proteins that localize to $\mathrm{T}$ tubules or the sarcoplasmic reticulum and that are involved in calcium release (ryanodine receptor 1 and 3; inositol 1,4,5-triphosphate 
receptor, type 1 , type 2 and type 3 ) or reuptake (sarco-endoplasmic reticulum transporting $\mathrm{Ca}^{2+}$ ATPase 1, 2 and 3). There was sufficient biopsy material available for analysis of gene ex- pression from 57 patients (from all groups, except control 1).

We grouped the patients according to whether there was significant damage to muscle structure $(n=25)$ or no damage $(n=$

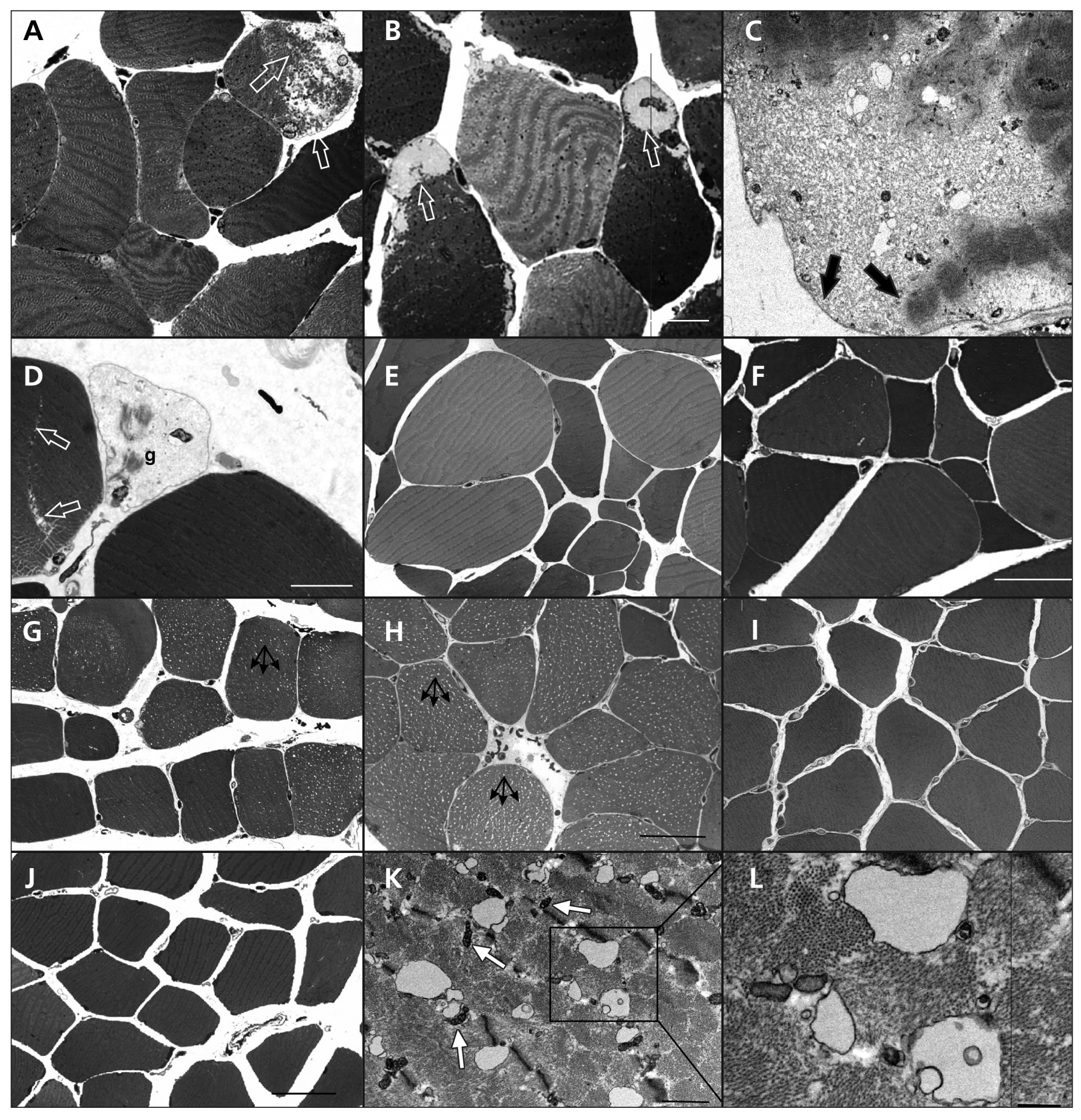

Figure 1: Skeletal muscle injury in patients with statin-associated myopathy. Light micrographs (A, B, D, E, F) and an electron micrograph (C) of transverse sections through the vastus lateralis muscle of patients with myopathy and current $(B, D, E, F)$ or previous $(A, C)$ statin use. Subsarcolemmal detachment of myofibrils is indicated by arrows in (A), (B) and (C). In (D), a ghost cell (g) is seen next to a developing split-fibre (arrows). (G, H): Light micrographs of semi-thin, transverse sections through the vastus lateralis muscle of myopathic patients currently $(\mathrm{G})$ or formerly taking statins $(\mathrm{H})$. Vacuolization is seen in most of the fibres (arrows). (I, J): The muscles fibres from control patients show no vacuolization. (K, L): Electron micrographs of a transverse section through the vastus lateralis muscle from a patient with myopathy who formerly used statins; intracellular vacuoles are present. The boxed area in (K) is enlarged in (L). The T-tubules are grossly vacuolated, but the adjacent mitochondria $(\mathrm{m})$ have a normal appearance. Bars: $\mathrm{A}, \mathrm{B}=10 \mu \mathrm{m} ; \mathrm{C}=0.2 \mu \mathrm{m} ; \mathrm{D}=$ $3 \mu \mathrm{m} ; \mathrm{E}, \mathrm{F}=50 \mu \mathrm{m} ; \mathrm{G}, \mathrm{I}, \mathrm{J}=50 \mu \mathrm{m}, \mathrm{H}=30 \mu \mathrm{m}, \mathrm{K}=1 \mu \mathrm{m} ; \mathrm{L}=0.5 \mu \mathrm{m}$. 

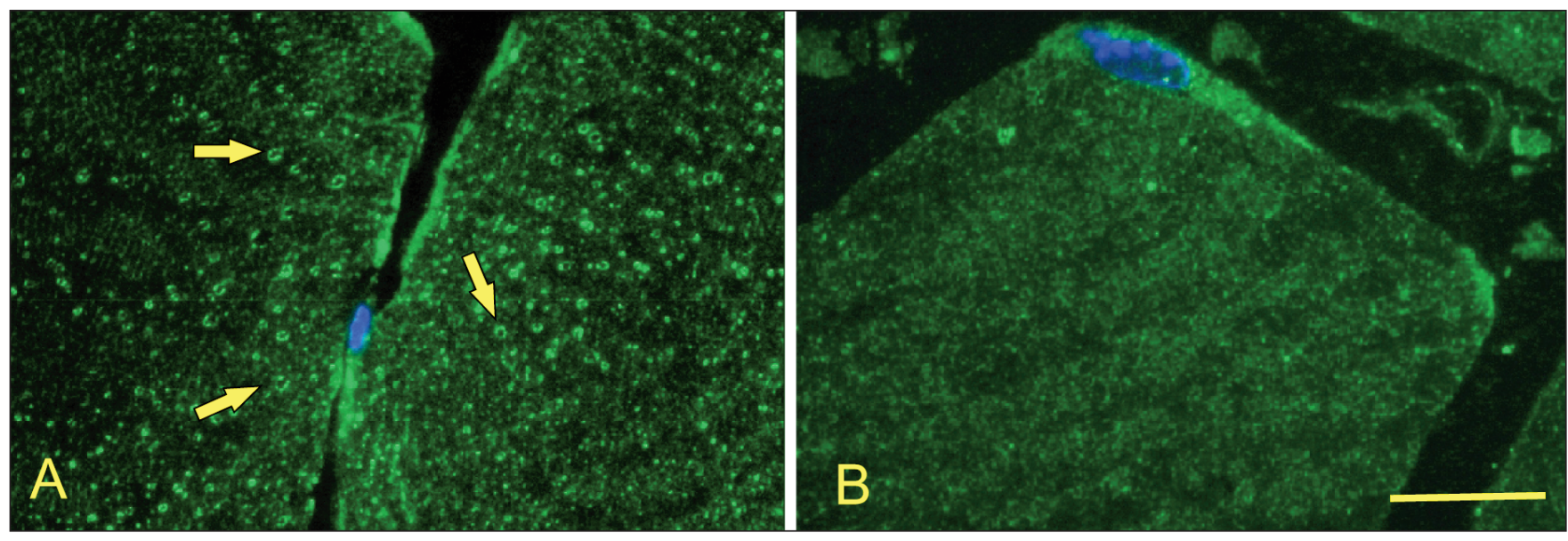

Figure 2: Fluorescence micrographs of transverse ultra-thin frozen sections labelled with a T-tubular marker (annexin A6, green) and a nuclear marker (Hoechst, blue) showing vacuolization (arrows) in a patient with statin-associated myopathy (A). The T-tubular system is undamaged in a control patient (B). Bar $=10 \mu \mathrm{m}$.

32). Of the patients with structural damage, 21 were currently taking stains. Thirteen of the patients without structural damage were taking statins. The expression of ryanodine receptor 3 mRNA was significantly higher among patients with structural damage than among those without damage (1.7, SEM 0.3,

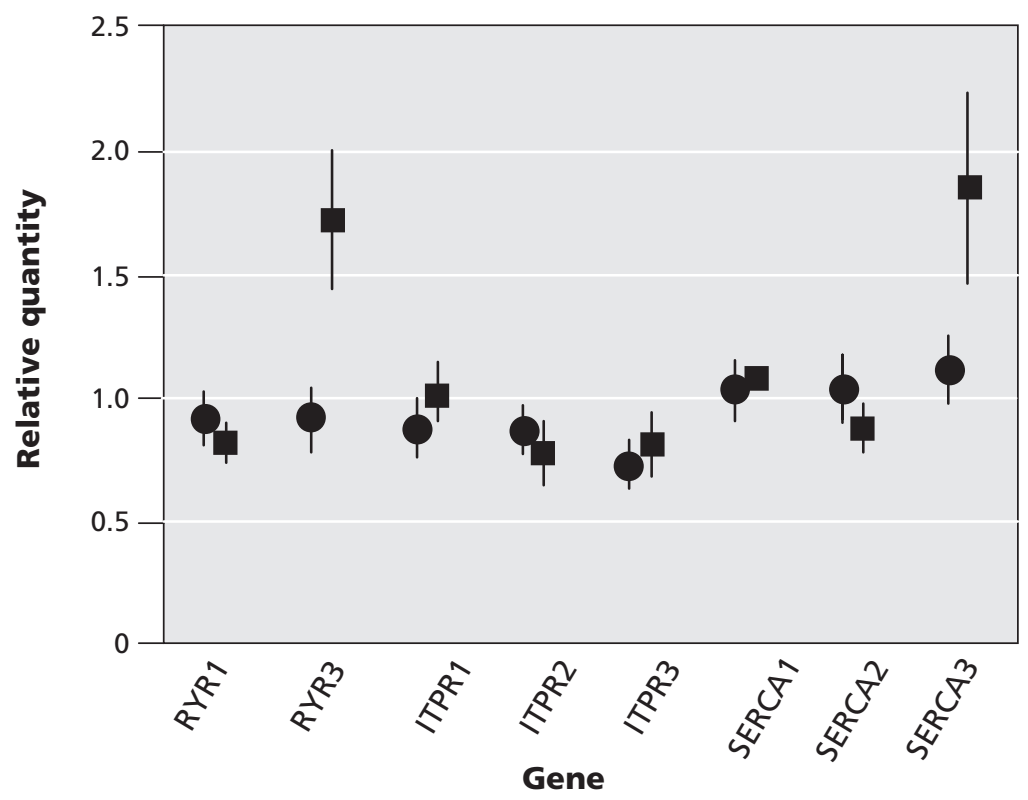

Figure 3: The levels of mRNA expression of 8 selected genes that code for components of calcium release or uptake. The expression of each gene was normalized to the amount of 18S RNA in the sample. Shown are the mean values with the standard error of the mean. Patients were grouped by the presence of significant muscle damage (squares, $n=25$ ) or no significant damage (circles, $n=32$ ). Note: RYR1 = ryanodine receptor 1 , RYR3 = ryanodine receptor 3 , ITPR1 = inositol 1,4,5-triphosphate receptor, type 1, ITPR2 = inositol 1,4,5triphosphate receptor, type 2, ITPR3 = inositol 1,4,5-triphosphate receptor, type 3; SERCA1 = sarco-endoplasmic reticulum transporting $\mathrm{Ca}^{2+}$ ATPase 1; SERCA2 = sarco-endoplasmic reticulum transporting $\mathrm{Ca}^{2+}$ ATPase 2; SERCA3 = sarco-endoplasmic reticulum transporting $\mathrm{Ca}^{2+}$ ATPase 3 . $p=0.039$; Figure 3). The expression of sarco-endoplasmic reticulum transporting $\mathrm{Ca}^{2+}$ ATPase 3 was also higher among those with structural damage than among those without damage (1.85, SEM 0.4, $p=0.51$; Figure 3$)$. This trend was not statistically significant, likely because of the high degree of variability of sarco-endoplasmic reticulum transporting $\mathrm{Ca}^{2+}$ ATPase 3 expression.

There were no differences in expression of the inositol triphosphate receptor isoforms (inositol 1,4,5-triphosphate receptor, type 1 $p=0.45$; inositol 1,4,5-triphosphate receptor, type $2 p=0.17$; inositol 1,4,5-triphosphate receptor, type $3 p=0.99)$, ryanodine receptor 1 $(p=0.724)$ and sarco-endoplasmic reticulum transporting $\mathrm{Ca}^{2+}$ ATPase pumps 1 and 2 (sarcoendoplasmic reticulum transporting $\mathrm{Ca}^{2+} \mathrm{AT}$ Pase $1 p=0.35$; sarco-endoplasmic reticulum transporting $\mathrm{Ca}^{2+}$ ATPase $2 p=0.63$ ) between patients with and without structural damage (Figure 3).

\section{Creatine phosphokinase}

The mean level of creatine phosphokinase was higher in patients with myopathy currently taking statins than in the other groups. With the exception of 1 patient who had rhabdomyolysis, the levels of creatine phosphokinase in the most patients with myopathy did not exceeded 1950 U/L (Figure 4). On the basis of the level of creatine phosphokinase alone, we could not distinguish between patients who had and those who had not taken statins (Figure 4).

\section{Interpretation}

We found a high prevalence of muscle injury in patients with clinically evident statin-associated myopathy. In addition, we identified a typical histo- 
pathological appearance of statin-associated myopathy, characterized by vacuolization of the T-tubular system with intact sarcolemma. This damage can occur without increased levels of circulating creatine phosphokinase. Although muscle symptoms typically improve rapidly after stopping statin therapy, our findings suggest that some patients are more susceptible to statinassociated myotoxicity and persistent structural injury. These findings have several important clinical implications.

We were surprised to find structural muscle injury in patients who had discontinued statin therapy for a considerable time. In general, cessation of treatment leads to alleviation of symptoms within days to weeks. ${ }^{15,16}$ However, because we identified patients on the basis of their history of statinassociated myopathy, we presumably selected for and identified a potentially vulnerable subgroup of patients who are prone to chronic structural lesions. We cannot exclude the possibility that these patients had an underlying myopathic process that was made evident by the use of statins.

Our findings call into question whether normal or mildly elevated levels of serum creatine phosphokinase can be used to exclude underlying and possibly ongoing muscle injury. Previous case reports have identified a small number of cases of statin-associated myopathy and pointed out the lack of a correlation between clinical symptoms and circulating levels of creatine phosphokinase. ${ }^{2,15,16}$ Our study adds to the previous observations by including larger numbers of patients and providing structural data from muscle biopsies.

In our study, the patients were taking a variety of statins, and all were given only moderate doses. We did not observe a correlation between higher doses of statins and a greater degree of muscle injury, nor did we see differences among the types of statins. No patient in our study was taking a high dose of statins (> $80 \mathrm{mg} /$ day), which is often recommended. ${ }^{17,18}$ The risk of statin-associated myotoxic adverse effects is enhanced by concomitant use of some medications. All statins are biotransformed in the liver, and drugs that interfere with this process may raise or lower the levels of these products in the blood. ${ }^{11-14}$ Fibrates, warfarin, macrolid antibiotics, antifungal agents and immunosuppressants all predispose patients to myopathy. ${ }^{11-14}$ In our study, there was no correlation between the extent of muscle damage and the use of any other single drug with a statin. However, the number of patients in each subgroup was small, and none of the patients were taking fibrates.

Glucocorticoids are known to cause wasting of proximal skeletal muscles. In the prestatin era, a study from our laboratory showed ultrastructural changes in the skeletal muscle of renal-transplant patients taking immunosuppressants and who had steroid-induced myopathy. ${ }^{19}$ Although a diffuse decrease in myofibrils was observed in these patients, neither intracellular vacuolation nor subsarcolemmal detach- ment of myofibrils, as was observed in our current study, were seen. ${ }^{19}$

We found that damage to the muscle fibres is largely restricted to the intracellular space, involving the T-tubular system. The intact lateral sarcolemma likely prevents leakage of creatine phosphokinase into the bloodstream. Vacuolization may be associated with increased vulnerability of the muscle fibre to mechanical injury. ${ }^{20}$ We have previously reported microscopic damage to skeletal muscle in asymptomatic patients taking statins, ${ }^{21}$ suggesting that statins may contribute to muscle damage. In our previous study involving asymptomatic patients, the characteristics of the damage were similar to that seen in patients with myopathy in the present study. However, the severity and prevalence of damage was considerably less in our previous study than that observed in the current population with myopathy and was well below our significance cutoff of $2 \%$ of fibres damaged. In the current study, only 1 asymptomatic patient had structural muscle damage greater than $2 \%$.

Ryanodine receptor 3 is the predominant isoform of this receptor in fetal and neonatal skeletal muscle. ${ }^{22}$ Adult skeletal muscle always contains ryanodine receptor 1 , and it may have variable amounts of ryanodine receptor $3 .^{23}$ The mechanistic and diagnostic implications of our finding of increased expression of ryanodine receptor 3 expression remains to be determined. First, it is unclear whether the expression of ryanodine receptor 3 was upregulated before statin use or whether its expression was increased as a result of statin-induced mus-

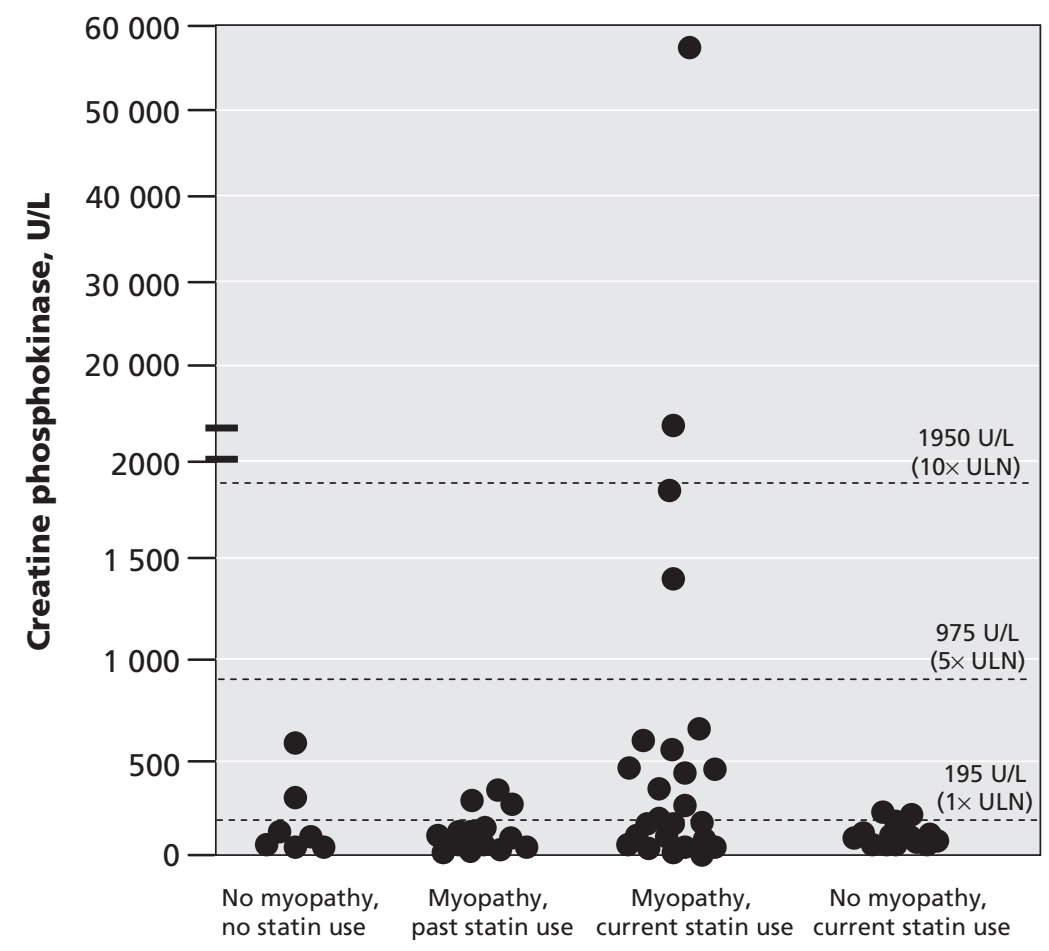

Figure 4: Circulating levels of creatine phosphokinase in patients with statinassociated myopathy and current (myopathy current statin) or past (myopathy former statin) statin therapy and in age-matched patients currently using statins with no myopathy. Note: ULN = upper limit of normal. 
cle injury. If the expression was increased before development of statin-induced myopathy, this could have important implications. Increased expression of ryanodine receptor 3 could represent a potential defect in calcium homeostasis, which could result in myofibre damage in statin users. Thus, the overexpression of ryanodine receptor 3 could contribute to the skeletal muscle selectivity of statin-associated myopathy, because cardiac muscle expresses ryanodine receptor 2 but not ryanodine receptor 3 .

\section{Limitations}

Our study has several limitations. First, patients were identified clinically as having statin-associated myopathy without clear criteria for what constitutes this clinical entity. In addition, these patients were not identified in a systematic manner. Therefore, the prevalence of underlying muscle damage among patients using statins and those with muscle-related complaints is unknown.

The small number of patients included in our study is also a limitation. However, we have included the largest series of cases studied by muscle biopsy. The small population size diminishes the power of this study to identify differences among patient groups and does not allow us to perform multivariable analyses. Thus, potential confounding factors could not be identified. The lack of longitudinal follow-up of these patients precludes us from determining what proportion of these patients will have their structural abnormalities resolve over time or with longer periods of cessation of statin therapy.

It also is likely that we underestimated the extent of muscle damage because all biopsies were taken from the vastus lateralis muscle, regardless of where the patient reported pain. Thus, it is possible that performing a biopsy of the affected muscle might have resulted in a higher prevalence of damage.

\section{Conclusion}

In current clinical practice, patients who present with muscle symptoms while receiving statin therapy have their creatine phosphokinase levels measured. If the level is within normal limits or is modestly elevated (current recommendations include creatine phosphokinase $<1950 \mathrm{U} / \mathrm{L}^{6}$ ), patients are frequently advised to continue their current statin therapy. This is based on the assumption that a lack of increased creatine phosphokinase levels supports a lack of underlying muscle damage. Our findings suggest that normal or moderately elevated levels of creatine phosphokinase do not exclude statinassociated muscle injury. Thus, alternative treatment strategies for patients with muscle symptoms need to be evaluated.

This article has been peer reviewed.

Competing interests: Richard Karas has received honoraria from Merck. Annette Draeger received honoraria from Pharmaceutical Product Development from January to April 2008 for diagnostic counselling in a phase 1 study unrelated to this article.

No competing interests declared by Markus Mohaupt, Eduard Babiychuk, Verónica Sanchez-Freire, Katia Monastyrskaya, Lakshmanan Iyer, Hans Hoppeler or Fabio Breil.
Contributors: All of the authors contributed to the study design, developed the protocol and contributed to the drafting of the manuscript. Verónica SanchezFreire, Lakshmanan Iyer and Richard Karas provided statistical knowledge. Markus Mohaupt and Hans Hoppeler performed the muscle biopsies. Richard Karas contributed specific clinical knowledge. Annette Draeger conceived the study and drafted the manuscript. All of the authors revised it for intellectual content and approved the final version submitted for publication.

Acknowledgements: We thank Catherine Allemann and Andrea Hostettler for technical assistance.

Funding: Financial support was received from the Swiss National Science Foundation's National Research Programme NRP 53 "Musculoskeletal Health-Chronic Pain" (grant 405340-104679/1 to A.D.) and from Swiss National Science Foundation grants (320000-111778 to K.M. and 3100A0-121980/1 to E.B.).

\section{REFERENCES}

1. Bruckert E, Hayem G, Dejager S, et al. Mild to moderate muscular symptoms with high-dosage statin therapy in hyperlipidemic patients - the PRIMO study. Cardiovasc Drugs Ther 2005;19:403-14.

2. Sinzinger H, Wolfram R, Peskar BA. Muscular side effects of statins. $J$ Cardiovasc Pharmacol 2002;40:163-71.

3. Thompson PD, Clarkson PM, Rosenson RS. An assessment of statin safety by muscle experts. Am J Cardiol 2006;97:69C-76C.

4. Thompson PD, Clarkson P, Karas RH. Statin-associated myopathy. JAMA 2003;289:1681-90

5. Armitage J. The safety of statins in clinical practice. Lancet 2007;370:1781-90.

6. Pasternak RC, Smith SC Jr, Bairey-Merz CN, et al. ACC/AHA/NHLBI clinical advisory on the use and safety of statins. Circulation 2002;106:1024-8.

7. Geiser J, Vogt M, Billeter R, et al. Training high-living low: changes of aerobic performance and muscle structure with training at simulated altitude. Int $J$ Sports Med 2001;22:579-85.

8. Bergström J. Percutaneous needle biopsy of skeletal muscle in physiological and clinical research. Scand J Clin Lab Invest 1975;35:609-16.

9. Zaugg P, Djonov V, Füchtbauer EM, et al. Sorting of murine vascular smooth muscle cells during wound healing in the chicken chorioallantoic membrane. Exp Cell Res 1999;253:599-606.

10. Draeger A, Monastyrskaya K, Burkhard FC, et al. Membrane segregation and downregulation of raft markers during sarcolemmal differentiation in skeletal muscle cells. Dev Biol 2003;262:324-34.

11. Evans M, Rees A. The myotoxicity of statins. Curr Opin Lipidol 2002;13:415-20.

12. Bellosta S, Paoletti R, Corsini A. Safety of statins: focus on clinical pharmacokinetics and drug interactions. Circulation 2004;109:III50-7.

13. Bottorff MB. Statin safety and drug interactions: clinical implications. Am J Cardiol 2006;97:27C-31C.

14. Harper CR, Jacobson TA. The broad spectrum of statin myopathy: from myalgia to rhabdomyolysis. Curr Opin Lipidol 2007;18:401-8.

15. Phillips PS, Haas RH, Bannykh S, et al. Statin-associated myopathy with normal creatine kinase levels. Ann Intern Med 2002;137:581-5.

16. Sinzinger H, O'Grady J. Professional athletes suffering from familial hypercholesterolaemia rarely tolerate statin treatment because of muscular problems. Br J Clin Pharmacol 2004;57:525-8.

17. O'Keefe JH Jr, Cordain L, Harris WH, et al. Optimal low-density lipoprotein is 50 to $70 \mathrm{mg} / \mathrm{dl}$ : lower is better and physiologically normal. J Am Coll Cardiol 2004;43:2142-6.

18. Nissen SE, Tuzcu EM, Schoenhagen P, et al. Effect of intensive compared with moderate lipid-lowering therapy on progression of coronary atherosclerosis: a randomized controlled trial. JAMA 2004;291:1071-80.

19. Horber FF, Hoppeler H, Herren D, et al. Altered skeletal muscle ultrastructure in renal transplant patients on prednisone. Kidney Int 1986;30:411-6.

20. Krolenko SA, Lucy JA. Vacuolation in T-tubules as a model for tubular-vesicular transformations in biomembrane systems. Cell Biol Int 2002;26:893-904.

21. Draeger A, Monastyrskaya K, Mohaupt M, et al. Statin therapy induces ultrastructural damage in skeletal muscle in patients without myalgia. J Pathol 2006;210:94-102.

22. Rossi D, Murayama T, Manini I, et al. Expression and functional activity of ryanodine receptors (RyRs) during skeletal muscle development. Cell Calcium 2007;41:573-80.

23. Engel AG, Franzini-Armstrong C. Myology. 4th ed. Toronto (ON): McGraw-Hill; 2004. p. 251.

Correspondence to: Dr. Annette Draeger, Institute of Anatomy, University of Bern, Baltzerstr. 2, 3012 Bern Switzerland; fax 413163 13807; draeger@ana.unibe.ch 